\title{
ORIGINAL RESEARCH \\ $\checkmark$ COMPARATIVE EFFECT OF CARPAL BONE MOBILIZATION VERSUS NEURAL MOBILIZATION IN IMPROVING PAIN, FUNCTIONAL STATUS AND SYMPTOMS SEVERITY IN PATIENTS WITH CARPAL TUNNEL SYNDROME
}

\author{
${ }^{1}$ Vikranth .G .R \\ ${ }^{2}$ Vinod Kumar .K .C \\ ${ }^{3}$ Lawrence Mathias
}

\section{ABSTRACT}

Background: Carpal tunnel syndrome (CTS) is a constellation of symptoms associated with compression of the median nerve at the wrist in carpal tunnel. The Purpose of this study is to find the comparative effective of carpal bone mobilization and neural mobilization in improving pain, Functional Status and Symptom Severity in patients with CTS.

Method: An experimental study design, 30 subjects with carpal tunnel syndrome were randomized into 2 groups with 15 subjects each in Group A and Group B. Subjects in Group A received carpal bone mobilization and subjects in Group B received median nerve mobilization. The duration of intervention was for two weeks. Outcome measurements such as pain using VAS, The Functional Status Score (FSS) and Symptom Severity Score (SSS) using the Boston's questionnaire for CTS were measured before and after two weeks of intervention.

Results: Analysis using paired ' $\mathrm{t}$ ' test found that there is a statistically significant improvement $(\mathrm{p}<0.05)$ in pain, Functional Status score and Symptom Severity score within the groups. Comparative analysis using independent ' $t$ ' test found that there is no statistically significant difference in improving pain, Functional Status score and Symptom Severity score between both the groups.

Conclusion: It is concluded that median nerve mobilization and carpal bone mobilization shown to be effective on improving pain, Functional Status and Symptom Severity in the treatment of patients presenting with carpal tunnel syndrome. However there is no significant difference in improvements obtained between the neural mobilization and carpal bone mobilisation.

Key words: Carpal tunnel syndrome, neurodynamics, neural mobilization, carpal bone mobilization, pain, Functional Status, Symptom Severity, Functional disability.

Received 09 ${ }^{\text {th }}$ May 2015, revised $17^{\text {th }}$ May 2015, accepted $02^{\text {nd }}$ June 2015

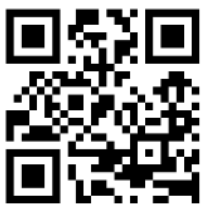

WwW.ijphy.org
DOI: 10.15621/ijphy/2015/v2i3/67025

\section{CORRESPONDING AUTHOR}

\footnotetext{
${ }^{2}$ Associate Professor in Physiotherapy, HOSMAT College of Physiotherapy,

Bangalore.

${ }^{3}$ Professor, Department of Orthopedics, K. S. Hegde Medical Academy, Mangalore Karnataka, India.
}

\section{${ }^{1}$ Vikranth .G .R, MPT}

Associate Professor in Physiotherapy, HOSMAT College of Physiotherapy, Bangalore, India. 


\section{INTRODUCTION}

Carpal tunnel syndrome (CTS) is a constellation of symptoms associated with compression of the median nerve at the wrist in carpal tunnel. ${ }^{1}$ CTS is the most common compression neuropathy, ${ }^{2}$ estimated to occur in $3.8 \%$ of the general population with a higher prevalence rate in women (3\%-5.6\%) than men (0.6\%-2.8\%). ${ }^{3-4}$ Women suffer more from CTS than men with a ratio of $3: 1$ between the ages of 25-55 years. ${ }^{3,5}$ A prevalence study done in India among laboratory workers also found prevalence rates of $21.5 \%$ which suggests that work pattern like repetitive use of hand makes a great contribution to the development of this condition. ${ }^{6}$

The main symptoms of CTS are numbness, pain and tingling of the first three fingers and radial side of the ring finger, nocturnal awakening is due to pain, numbness and impaired fine motor control because of weakness of the hand. In moderate to severe cases there will be hand clumsiness, weakness of thumb and atrophy of thenar muscles may be seen which will eventually decrease the functional ability of the hand and interfere with daily activities. ${ }^{2,7}$

Physiotherapy methods for treating CTS such as electrotherapy may provide some symptomatic relief. However, such methods do not address the pathological neurodynamics of the median nerve and its surrounding structures. Anecdotal clinical evidence supports physiotherapeutic intervention with these patients as improvement has been seen in response to a variety of manual therapy treatment approaches. There is also some evidence of chiropractic or osteopathic manual intervention providing some relief of symptoms for patients experiencing CTS..$^{8-13}$

Several authors have suggested that patients treated with manipulation of carpal bones i.e. scaphoid and pisiform along with stretching of flexor retinaculum is helpful in treating CTS. Literature concerning the effects of joint mobilization as applied by manual therapists is however lacking and at present there is no specific literature exploring the treatment of CTS. At the same time some studies have investigated the effects of nervous system mobilization on nerve entrapment problems stating that neural mobilization is helpful in improving axonal transport and by this mechanism to improve nerve conduction. $^{8-13}$ Also, neural mobilization may reduce the pressure existing within the nerve and could therefore result in an improvement of blood flow to the nerve. ${ }^{10}$ Consequently, regeneration and healing of an injured nerve may also occur.
As there is limited literature available in the management of CTS there are no studies clearly defining which mode of management is better than the other to our knowledge. Therefore this study with research question 'does there is a difference in effect of neural mobilization and carpal bone mobilization for the treatment of CTS? As there are no studies available to compare the effectiveness of neural mobilization and carpal bone mobilization for the treatment of CTS, hence purpose of this study is to find the comparative effective of carpal bone mobilization and neural mobilization in improving pain Functional Status and Symptom Severity in patients with CTS. It was null hypothesized that there will be no significant difference between carpal bone mobilization and neural mobilization in improving pain, Functional Status and Symptom Severity in patients with CTS.

\section{METHODOLOGY}

An experimental comparative study design with two groups- Group A and Group B. As this study involved human subjects the Ethical Clearance was obtained from the Ethical Committee of The Oxford College of Physiotherapy, Bangalore as per the ethical guidelines of Bio-medical research on human subjects. Subjects included who were diagnosed with carpal tunnel syndrome aged 25-55 years of both male and female, with positive phalen's test, Positive tinel's test, Positive ULTT2, ${ }^{12-}$ ${ }^{15}$ VAS above or equal to 4, Subjects who are willing to participate in the study. ${ }^{16}$ Subjects were excluded with Psycho-social problem, Double crush syndromes, Cervical or thoracic origin of symptoms, medical conditions like uncontrolled Diabetes, Herpes Zoster, recent fractures crush injuries, shoulder injuries, Scaphoid instability, Hypermobile joints, Pregnancy, Women under hormonal replacement therapy. Subjects were recruited from The Oxford Physiotherapy OPD, Ortho One Orthopedic Clinic Jayanagar, Sparsh Hospitals Bangalore.

The study was conducted at The Oxford Physiotherapy OPD, Bangalore. Subjects who meet inclusion criteria were recruited by Simple random sampling method using closed envelops, randomly allocated subjects into two groups. Subjects who meet inclusion criteria, total 30 Subjects $(n=30), 15$ in each group, were informed about the study and a written informed consent was taken. The duration of intervention in the study was 2 weeks.

Procedure of intervention for Group A: Subjects in this group were received carpal Bane Mobilization. ${ }^{12,13}$ The subjects was made to sit on a chair. The therapist in standing position grasps the subject's hand, so the elbow hangs unsupported. 
The weight of the arm provides slight distraction to the joint (Grade 1) and then applies the glides. The therapist identifies the scaphoid which is to be mobilized and place the index fingers on the volar surface of the bone to be stabilized. Place the overlapping thumbs on the dorsal surface of the scaphoid. The rest of the fingers hold the subject's hand to be relaxed. In this the force is given from the overlapping thumbs on the dorsal surface. By mobilizing from dorsal surface pressure against the nerve, blood vessels and tendons in the carpal tunnel were minimized and thus stronger mobilizing force was given. Thus the therapist administred volar or dorsal glides to the scaphoid bone with an oscillation of 30-40 per minute. Along with this lateral glide of proximal row of carpal bones and flexor retinaculum stretching was also given. ${ }^{12,13}$ Grade of mobilization and progression of treatment was decided on the subject's irritability and severity. This treatment was administered for 5 times a week for a total period of 2 weeks. Pre and post scores of pain and disability were taken on the $1^{\text {st }}$ day and at the end of $2^{\text {nd }}$ week respectively.

Procedure of intervention for Group B: Subjects in this group received Neural Mobilization for the Median nerve. ${ }^{10,14}$ The subjects were made to lie down on a couch in supine position. The neural mobilization technique was applied as described by Butler i.e. ULTT2. It was performed as the subject lies slight diagonally across the bed with the head towards the affected side and the scapula of the affected side free of the bed. The therapist thigh rests against the subject's affected shoulder. The therapist distal hand holds the subject's elbow and proximal hand holds the wrist or to the point where symptoms were produced. Then the subject's shoulder is depressed carefully, in this position it is possible to look at the subject's face to pick up any non verbal information.

Then the shoulder is abducted to 10 degree so that the arm is parallel to the side of the bed. The shoulder depression is maintained and subsequently extends the subjects elbow followed by lateral rotation of the shoulder. Then the therapist thumb was slipped in the web space between the subject's thumb and index finger. The therapist then extends the subject's wrist, fingers and thumb. Then for sensitizing additional degree of shoulder abduction is attained. All the movements are taken to the end of the available range. Once a symptom has been reproduced, if more distal, then shoulder depression can be released and the effects assessed. If more proximal, the wrist was moved to assess if there are any alterations in the reproduced symptoms. These neural stretches were held for 10 seconds and were repeated 10 times per session. This treatment was administered for 5 times a week for a total period of 2 weeks. Pre and post scores of pain and disability were taken on the $1^{\text {st }}$ day and at the end of $2^{\text {nd }}$ week respectively.

\section{OUTCOME MEASURES}

Pain was measured with the visual analog scale where subjects were asked to indicate on the scale, the severity of pain from the range of 0 (zero) no pain to 10 (ten) most severe pain was measured. ${ }^{17}$

The Functional Status Score (FSS) and Symptom Severity Score (SSS) were measured using the Boston's questionnaire for CTS and asked to answer the questions appropriately according to their symptoms and functional status. ${ }^{18}$

Pre treatment scores were taken from the subjects, which included assessment of pain using visual analog scale (VAS), and symptoms and functional status using Boston's questionnaire for carpal tunnel syndrome. After completion of treatment period of 2 weeks, post treatment scores were taken to find out the difference between the pre and post treatment scores.

\section{Statistical Methods}

Descriptive statistical analysis was carried out in the present study. Out Come measurements analyzed are presented as mean \pm SD. Significance is assessed at $5 \%$ level of significance with $p$ value was set at 0.05 less than this is considered as statistically significant difference. Paired ' $t$ ' test as a parametric and Wilcoxon signed rank test as a non-parametric test have been used to analysis the variables pre-intervention to post-intervention with calculation of percentage of change. Independent ' $\mathrm{t}$ ' test as a parametric and Mann Whitney U test as a non-parametric test have been used to compare the means of variables between two groups with calculation of percentage of difference between the means. The Statistical software namely SPSS 16.0, Stata 8.0, MedCalc 9.0.1 and Systat 11.0 were used for the analysis of the data and Microsoft word and Excel have been used to generate graphs, tables etc.

\section{RESULTS}

The study was completed with total 30 subjects (Table-1). In Group A there were 15 subjects with mean age $40.73 \pm 9.86$ years and there were 5 males 10 females were included in the study. In Group B there were 15 subjects with mean age $40.33 \pm 9.41$ years and there were 8 males 7 females were included in the study. There is no significant difference in mean ages between the groups. 
Pre to Post test within group analysis found that (Table-2,3,4) there is a statistically significant change in means of VAS, Functional Status Score (FSS) and Symptom Severity Score (SSS) when means were analyzed from pre intervention to post intervention within the groups with $p<0.000$.
Comparative Analysis between the group Pre and Post test means found that (Table-2,3,4) there is no statistically significant change in means of VAS, FFF, SSS when means were analyzed between the groups with $p<0.000$.

Table 1: Basic Characteristics of the subjects studied

\begin{tabular}{|c|c|c|c|c|c|c|}
\hline \multicolumn{2}{|c|}{$\begin{array}{c}\text { Basic Characteristics of the subjects } \\
\text { studied }\end{array}$} & \multicolumn{2}{|c|}{ Group A } & \multicolumn{2}{|c|}{ Group B } & $\begin{array}{l}\text { Between the groups } \\
\text { Significance }\end{array}$ \\
\hline \multicolumn{2}{|c|}{ Total number of subjects studied (n) } & \multicolumn{2}{|c|}{15} & \multicolumn{2}{|c|}{15} & -- \\
\hline \multicolumn{2}{|c|}{$\begin{array}{l}\text { Age in years } \\
(\text { Mean } \pm \text { SD) }\end{array}$} & \multicolumn{2}{|c|}{$40.73 \pm 9.86$} & \multicolumn{2}{|c|}{$40.33 \pm 9.41$} & $\mathrm{p}=0.910(\mathrm{NS})$ \\
\hline \multirow{2}{*}{ Gender } & Males & $\mathrm{n}=5$ & $33.3 \%$ & $\mathrm{n}=8$ & $55.3 \%$ & \multirow{2}{*}{$\mathrm{p}=0.269(\mathrm{NS})$} \\
\hline & Females & $\mathrm{n}=10$ & $66.7 \%$ & $\mathrm{n}=7$ & $46.7 \%$ & \\
\hline
\end{tabular}

Table 2: Comparative analysis of VAS score between two groups

\begin{tabular}{|c|c|c|c|}
\hline VAS & Group A & Group B & P value \\
\hline Pre & $5.53 \pm 1.25$ & $5.60 \pm 1.40$ & $0.892(\mathrm{NS})$ \\
\hline Post & $4.07 \pm 1.39$ & $3.67 \pm 1.23$ & $0.411(\mathrm{NS})$ \\
\hline Difference & 1.48 & 1.93 & - \\
\hline P value & $<0.001^{* *}$ & $<0.001^{* *}$ & - \\
\hline
\end{tabular}

** Statistically Significant difference p < 0.05; NSNot significant

Graph 1: Comparative analysis of VAS score between two groups

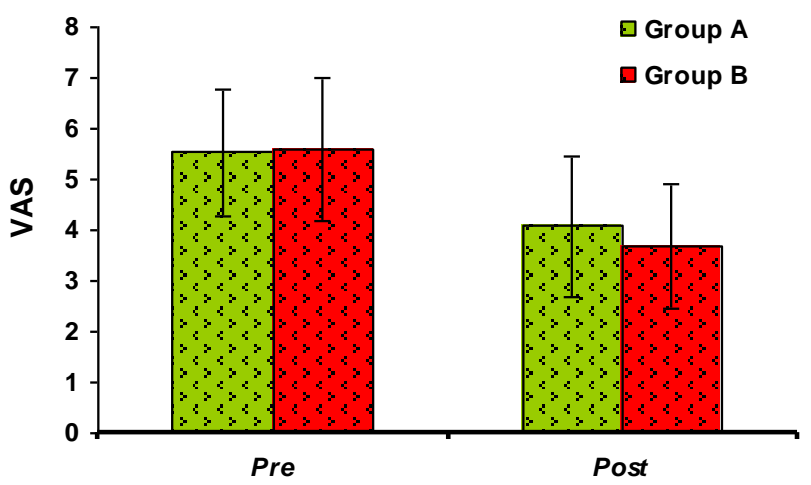

Table 3: Comparative analysis of FSS between two groups

\begin{tabular}{|c|c|c|c|}
\hline FSS & Group A & Group B & P value \\
\hline Pre & $2.32 \pm 0.79$ & $2.56 \pm 0.78$ & 0.413 (NS) \\
\hline Post & $1.96 \pm 0.70$ & $2.05 \pm 0.69$ & 0.712 (NS) \\
\hline Difference & 0.36 & 0.50 & - \\
\hline P value & $<0.001^{* *}$ & $<0.001^{* *}$ & - \\
\hline
\end{tabular}

** Statistically Significant difference $p<0.05$; NSNot significant
Graph 2: Comparative analysis of FSS between two groups

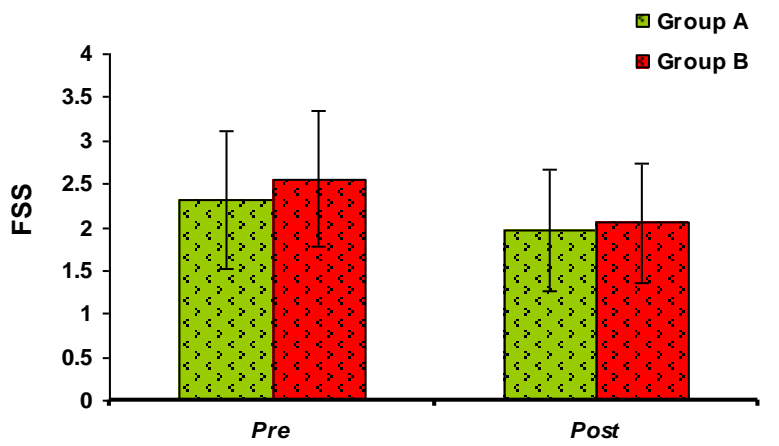

Table 4: Comparative analysis of SSS between two groups

\begin{tabular}{|c|c|c|c|}
\hline SSS & Group A & Group B & P value \\
\hline Pre & $2.48 \pm 0.56$ & $2.56 \pm 0.62$ & 0.729 (NS) \\
\hline Post & $2.07 \pm 0.56$ & $2.03 \pm 0.46$ & 0.809 (NS) \\
\hline Difference & 0.40 & 0.53 & - \\
\hline P value & $<0.001^{* *}$ & $<0.001^{* *}$ & - \\
\hline ** Statistically Significant difference p<0.05; NS- \\
Not significant
\end{tabular}

Graph 3: Comparative analysis of SSS between two groups

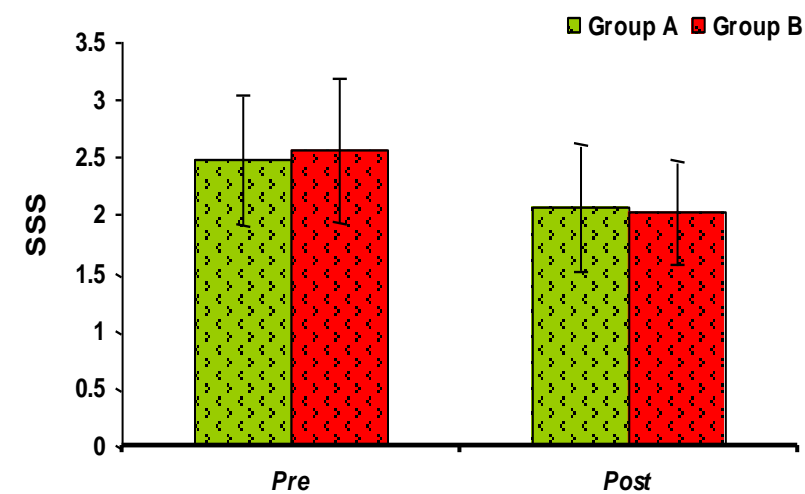


Table 5: Comparison of pre and post within the group mean difference between two groups

\begin{tabular}{|c|c|c|c|c|}
\hline & Group A & Group B & P value & $\begin{array}{c}\text { Effect } \\
\text { Size }\end{array}$ \\
\hline \begin{tabular}{c|c|c|c|}
$\Delta$ in \\
VAS
\end{tabular} & $1.47 \pm 0.99$ & $1.93 \pm 0.96$ & $\begin{array}{c}0.201 \\
(\mathrm{NS})\end{array}$ & $\begin{array}{c}0.46 \\
(\mathrm{~S}-\mathrm{M})\end{array}$ \\
\hline \begin{tabular}{c|c}
$\Delta$ in \\
FSS
\end{tabular} & $0.36 \pm 0.19$ & $0.50 \pm 0.19$ & $\begin{array}{c}0.052+ \\
(\mathrm{NS})\end{array}$ & $\begin{array}{c}0.72 \\
(\mathrm{M}-\mathrm{L})\end{array}$ \\
\hline $\begin{array}{c}\Delta \text { in } \\
\text { SSS }\end{array}$ & $0.40 \pm 0.25$ & $0.53 \pm 0.24$ & $\begin{array}{c}0.184 \\
(\mathrm{NS})\end{array}$ & $\begin{array}{c}0.52 \\
(\mathrm{M})\end{array}$ \\
\hline
\end{tabular}

$\Delta$ Pre and post within the group mean difference; NS- Not significant

Graph 4: Comparison of pre and post within the group VAS mean difference between two groups

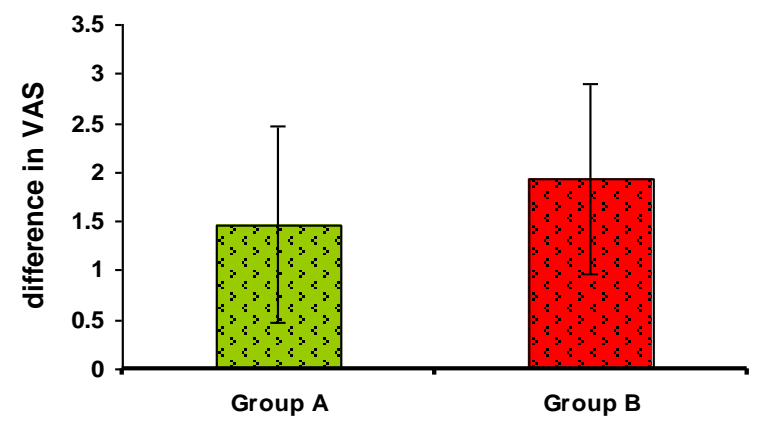

Graph 5: Comparison of pre and post within the group FSS mean difference between two groups

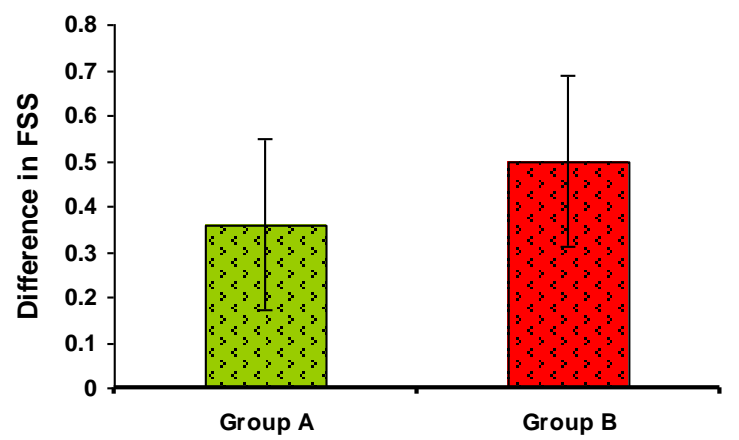

Graph 6: Comparison of pre and post within the group SSS mean difference between two groups

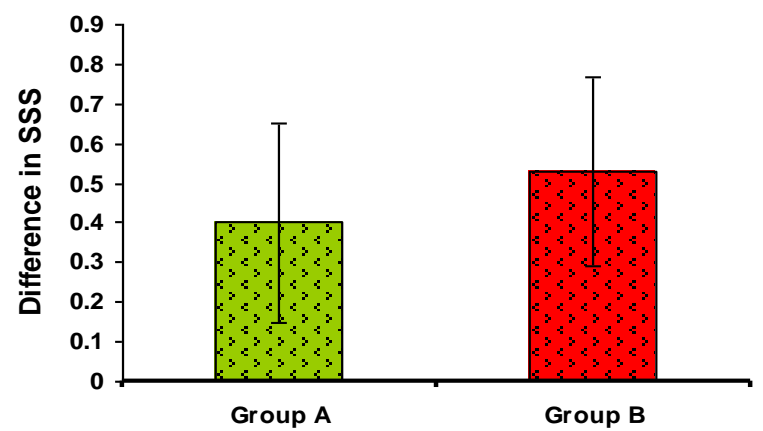

\section{DISCUSSION}

This study examined the results of carpal bone mobilization and neural mobilization in patients who had CTS. In the present study 30 subjects were taken and divided into two groups. Group-A was given carpal bone mobilisation and Group-B was given neural mobilisation. In this study the effectiveness of carpal bone mobilisation in reducing pain, Functional Status Score (FSS) and Symptom Severity Score (SSS) in patients with CTS and the effectiveness of neural mobilisation in reducing pain, Functional Status Score and Symptom Severity Score in patients with CTS was studied and the comparison between carpal bone mobilisation and neural mobilisation is done. ${ }^{11-13}$

Carpal bone mobilisation has a significant effect in reducing pain, Functional Status Score and Symptom Severity Score in CTS. In table 2 the pre and post values of VAS are given. There is a difference of 1.48 in pre and post values which is showing the decrease in pain, in subjects after a treatment of carpal bone mobilisation of 2 weeks is seen. The improvement is significant with $p<0.001$. Tal - Akabi et al in 2000 had concluded in their study that carpal bone mobilisation is effective in reducing pain in CTS. In table 4 the Symptom Severity Score difference is calculated and it is of 0.36 and FSS in table 6 is 0.40 shows that there is a significant effect of carpal bone mobilisation is present in reducing disability in CTS. Tal- Akabi et al has also concluded that carpal bone mobilisation will reduce disability.

Neural mobilisation also had significant effect in reducing pain in CTS. The difference in VAS score in pre and post is 1.93 and Functional Status Score and Symptom Severity Score are of 0.5 and 0.53 which shows that there is a effect in decrease in pain and disability in CTS. Dimitrioskostoppolous in 2003 has done a study and concluded that neural mobilisation is effective in reducing pain and disability in CTS. ${ }^{8}$

The VAS score were found to be statistically significant having a difference of 1.48 in Group A and 1.93 in Group B with a p value of $<0.001$. But when comparing pre and post scores for Group A and Group B it was seen that differences in both the groups are not statistically significant.

FSS is compared between the groups and it is 0.36 in Group A and Group B is 0.50 with a p value p. $<0.001$ in this calculation the neural mobilisation is said to be more effective than carpal bone mobilisation. Rozmarynetal in1998 has done a study and told that neural mobilisation techniques are effective in CTS. A. Kalin et al in 2002 has tested 28 subjects of CTS and for which neural gliding exercises were given and concluded that these exercises have significant effect in patients with CTS. Richard fell is et al have done a systematic review and concluded a positive therapeutic benefit from using neural mobilization.

SSS has been compared between groups and the values are 0.4 and 0.53 . In this it is seen that neural 
mobilisation is clinically more effective than carpal bone mobilisation but according to statistical analysis there is no significant difference present.

From the above differences values of VAS, FSS and SSS it is stated that there is no significant difference present in between the groups and thus null hypothesis is accepted. From the above two interventions it is found that both the techniques i.e carpal bone mobilization and neural mobilisation is proved to be effective but when compared to each other there is no significant difference between the two i.e. neural mobilization and carpal bone mobilisation.

\section{LIMITATIONS}

- Sample size was small.

- Nerve conduction studies were not used in this study.

- Follow up was not done, so this study cannot comment on long term results.

\section{RECOMMENDATION}

- This study can be carried out in a more time efficient manner by choosing the appropriate parameters and treatment locations.

- A larger sample size is highly recommended to make the study more reliable.

- Duration of the study should be increased to see long term effects.

\section{Conclusion}

It is concluded that median nerve mobilization and carpal bone mobilization shown to be effective on improving pain, Functional Status and Symptom Severity in the treatment of patients presenting with carpal tunnel syndrome. However there is no significant difference in improvements obtained between the neural mobilization and carpal bone mobilisation.

This research has therefore demonstrated that patients experiencing CTS can improve after manual therapy, and therefore provides support for the use of manual therapy in the conservative management methods of treating patients with this condition with satisfactory results. However, more research needs to be carried out to further support these findings.

\section{Acknowledgement}

Authors were expressing their sense of gratitude's to the people who helped and encouraged them for the guidance and completion of this study.

Conflicts of interest: None

\section{REFERENCES}

1. Steves JC. The electrodiagnosis of carpal tunnel syndrome, revised. Rochester (MN). American association of electrodiagnostic medicine 1997. John Wiley \& Sons, Inc. Muscle Nerve 1997;20(12):1477-1486.

2. David M. Dawson. Entrapment Neuropathies of the Upper Extremities. N Engl J Med. 1993; 329: 2013-2018.

3. IsamAtroshi, Christina Gummersson, ragnarJhnsson, Edwald Ornstein, Jonas Ranstam, Ingmar Rosen. Prevalence of CTS in a general population. JAMA.1999;282(2):153158.

4. Hagberg M, Morgenstern H, Kelsh M. Impact of occupation on the prevalence of carpal tunnel syndrome. Scand J work Environ Health. 1992;18(6):337-345.

5. Macfarlane GJ. Identification and prevention of work- related CTS. The Lancet. 2001; 357, (9263), 1146-1147

6. Seema V. Kamarddi, Latti RG, Kodliwadmath MV. Determination of the prevalence of CTS in the laboratory workers of Jawaharlal Nehru Medical College, Belgaum. A cross sectional study. AJEBS. 2010;1(4):740-746.

7. Mackinnon SE. Pathophysiology of nerve compression. Hand Clin. 2002; 18(2):231-241.

8. Dimitrious Kostopoulos. Treatment of carpal tunnel syndrome: a review of the non-surgical approaches with emphasis in neural mobilization. Journal of Body work and Movement Therapies. 2004;8(1):2-8.

9. Michelle L Heebner, Toni S Roddey. The effects of neural mobilization in addition to standard care in persons with carpal tunnel syndrome from a community hospital. J Hand Therapy. 2008;21(3) 229-40.

10. Butler D. Mobilization of the Nervous system.1st edn;1993

11. Maitland GD. Peripheral Manipulation. 3rd edn;1991.

12. Patterson MM. Manipulation can Stretch the Transverse Carpal Ligament. Journal of the American Osteopathic Association. 1998; 98(12): 662.

13. Sucher BM, Hinrichs RN. Manipulative Treatment of CTS, Biomechanical and Osteopathic Intervention. Journal of American Osteopathic Association. 1998; 98(12); 679-686.

14. Mulligan BR. Manual Therapy "NAGS", "SNAGS","PRP'S" etc. $2^{\text {nd }}$ edition;1992.

15. LeighannLitchr Kelly, Sharon A. Martino, Joan E. Broderick and Arthur A. Stone. A systemic review of measures used to assess chronic musculoskeletal pain in clinical and 
randomized controlled clinical trials. Journal of pain. 2007; 8(12):906-913.

16. Oonstra, Anne M.a, Schiphorst Preuper, Henrica R.b d, Reneman, Michiel F.b d, Posthumus, Jitze B.a, Stewart, Roy E. Reliability and validity of the visual analogue scale for disability in patients with chronic musculoskeletal pain. International Journal of Rehabilitation Research.2008; 31(2):165-169.

17. Jose C de Carvalho Leite, Christina JeroschHerold and Fujian Song. A systematic review of the psychometric properties of the Boston Carpal Tunnel Questionnaire. BMC Musculoskeletal Disorders 2006; 7:78.
18. LaJoie, A Scott, McCabe, Steven J, Thomas, Binu, Edgell, Stephen E. Determining the Sensitivity and Specificity of Common Diagnostic Tests for Carpal Tunnel Syndrome Using Latent Class Analysis. Plastic \& Reconstructive Surgery.2005; 116 (2):502-507.

19. Robert G. Marx, Claire Bombardier, James G. Wright. Kuschner SH, Ebramzadeh E, Johnson D, Brien WW, Sherman R. What do we know about the reliability and validity of physical examination tests used to examine the upper extremity? J Hand Surg. 1999; 24(1):185-193.

\section{Citation}

G.R, V., Kumar, V., \& Mathias, L. (2015). COMPARATIVE EFFECT OF CARPAL BONE MOBILIZATION VERSUS NEURAL MOBILIZATION IN IMPROVING PAIN, FUNCTIONAL STATUS AND SYMPTOMS SEVERITY IN PATIENTS WITH CARPAL TUNNEL SYNDROME. International Journal of Physiotherapy, 2(3), 524-530. 